\title{
Production of Amastigotes from Metacyclic Trypomastigotes of Trypanosoma cruzi
}

\author{
Víctor T Contreras ${ }^{+}$, María C Navarro, Ana R De Lima, Rosa Arteaga, Francy Duran, \\ José Askue*, Yunaimy Franco
}

Laboratorio de Protozoología, Centro BioMolP, Facultad de Ciencias de la Salud, Universidad de Carabobo, Bárbula, Valencia, Estado Carabobo, Venezuela *Facultad de Agronomía, Universidad Central de Venezuela, Maracay, Estado Aragua, Venezuela

Attempts to recreate all the developmental stages of Trypanosoma cruzi in vitro have thus far been met with partial success. It is possible, for instance, to produce trypomastigotes in tissue culture and to obtain metacyclic trypomastigotes in axenic conditions. Even though T. cruzi amastigotes are known to differentiate from trypomastigotes and metacyclic trypomastigotes, it has only been possible to generate amastigotes in vitro from the tissue-culture-derived trypomastigotes. The factors and culture conditions required to trigger the transformation of metacyclic trypomastigotes into amastigotes are as yet undetermined. We show here that pre-incubation of metacyclic trypomastigotes in culture (MEMTAU) medium at $37^{\circ} \mathrm{C}$ for $48 \mathrm{~h}$ is sufficient to commit the parasites to the transformation process. After $72 \mathrm{~h}$ of incubation in fresh MEMTAU medium, <90\% of the metacyclic parasites differentiate into forms that are morphologically indistinguishable from normal amastigotes. SDS-PAGE, Western blot and PAABS analyses indicate that the transformation of axenic metacyclic trypomastigotes to amastigotes is associated with protein, glycoprotein and antigenic modifications. These data suggest that (a) T. cruzi amastigotes can be obtained axenically in large amounts from metacyclic trypomastigotes, and $(b)$ the amastigotes thus obtained are morphological, biological and antigenically similar to intracellular amastigotes. Consequently, this experimental system may facilitate a direct, in vitro assessment of the mechanisms that enable T. cruzi metacyclic trypomastigotes to transform into amastigotes in the cells of mammalian hosts.

Key words: amastigogenesis - axenic conditions - differentiation - extracelullar amastigotes - morphogenesis

Trypanosoma cruzi, the causative agent of Chagas disease, is mainly transmitted to humans by reduviid insects. This parasite has a complex life cycle that alternates between invertebrate and vertebrate hosts. Transmission is initiated by insect vectors, which defecate after a blood meal and release metacyclic trypomastigotes near the bite wound. This infective stage is now capable of reaching an intracellular location and transforming into amastigotes. The amastigotes then divide and transform into trypomastigotes (called bloodstream trypomastigotes). Upon leaving the cell, the trypomastigotes enter the bloodstream, where they are eventually taken up by the insect host. In the intestine of the invertebrate host the blood trypomastigote transforms into epimastigotes. The epimastigotes divide and give rise to infective metacyclic trypomastigotes (Brener 1973). Consequently, natural T. cruzi amastigogenesis occurs when metacyclic trypomastigotes from triatomine urine differentiate into amastigotes inside mammalian host cells.

This research was supported by grants from Fonacit S197000664 (VTC, ARDL), Fonacit S1-2001000683 (MCN, VTC, ARDL), Codecih FCS-97018 (ARDL, VTC) and Codecih FCS-99010 (MCN, VTC).

${ }^{+}$Corresponding author. Present address: VLN 1500 PO Box 025685, Miami, Fl 33102-5685 USA. Fax: +58241-8673342.

E-mail: convictu@cantv.net.ve

Received 15 May 2002

accepted 21 August 2002
Detailed knowledge of the invasion mechanisms of vertebrate cells by $T$. cruzi has been obtained using cellculture-derived trypomastigotes that are equivalent to bloodstream trypomastigotes (Burgleigh \& Andrews 1995). These studies have revealed that following cell invasion, the trypomastigotes penetrate the host cells. The trypomastigote-to-amastigote transformation is thus initiated, and 1-2 $\mathrm{h}$ later, an acid-active membrane pore-forming protein combined with neuraminidase activity synergistically promote the escape of the trypomastigote from the parasitophorous vacuole into the host cell cytoplasm, where the differentiation into amastigote form is completed.

Using axenic conditions, several investigators have reported the morphological differentiation of bloodstream trypomastigotes into amastigotes and several factors stimulating this transformation have been described (Villalta \& Kierzenbaum 1982, Andrews et al. 1987, Kambara et al. 1990, Tomlinson et al. 1995). Some of these factors are well characterized and represent physiological stress for the parasites. Other authors have obtained extracelullar amastigotes from epimastigotes using highly enriched media supplemented with chicken plasma or vitamins, nucleotides and fetal bovine serum (FBS) (Kimura et al. 1978, Pan 1978, Rondinelli et al. 1988). Although metacyclic trypomastigotes of $T$. cruzi can be obtained from axenic culture (Chiari 1975, Contreras et al. 1985), it has not been possible to obtain amastigotes in axenic conditions from metacyclic trypomastigotes.

Little is known about the factors that control primary amastigogenesis. One obvious difference between a warm-blooded vertebrate and an invertebrate is tempera- 
ture, and this has been recognized as a morphogenetic element for parasitic protozoa that use arthropods as transmission vectors (Shapira et al. 1988). Since there is difference of approximately 10 degrees between the invertebrate and vertebrate hosts, it is likely that temperature may play an important role in triggering primary amastigogenesis in $T$. cruzi. For example, even though mammalian cells cultured at room temperature can interiorise metacyclic forms of T. cruzi, transformation of the parasite into amastigotes is not observed (Deane et al. 1984). However, other undetermined factors must exist as most reported experimental designs have failed to recapitulate T. cruzi's primary amastigogenesis (Tyler \& Engman 2001).

From a parasitological perspective, metacyclic trypomastigotes and bloodstream trypomastigotes appear to share similar biological properties. Both stages are capable of binding to the target cell's membrane, active penetration, transformation into amastigotes, intracellular multiplication and further differentiation into trypomastigotes. However, metacyclic forms and bloodstream trypomastigotes are known to display stage-specific antigens and actually display different modes of interaction with host cells (Burgleigh \& Andrews 1995). For instance, metacyclic trypomastigotes express several glycoproteins on their surface that interact with mammalian cells, and which have no counterpart in blood trypomastigotes (Ruiz et al. 1993). This difference in gene expression justifies the discrimination between bloodstream and metacyclic trypomastigotes as physiologically distinct life cycle stages (Tyler \& Engman 2001).

In our current work, we describe an experimental protocol for inducing the transformation of T. cruzi metacyclic trypomastigotes into amastigotes. We have studied the influence of both temperature and pre-incubation times on axenic metacyclics and have found that the incubation of parasites in MEMTAU medium at $37^{\circ} \mathrm{C}$ can induce amastigogenesis. Furthermore, we provide morphological, biological and antigenic evidence demonstrating that the in vitro-generated amastigotes are similar to intracellular amastigotes.

\section{MATERIALS AND METHODS}

Parasites and stages - Throughout this study, cloned EPm6 isolate of T. cruzi was used. The parasite was cloned and maintained by alternate triatomine/mouse passages as previously described (Contreras et al. 1994). Epimastigotes were cultured at $27^{\circ} \mathrm{C}$ in LIT medium, and purified metacyclic trypomastigotes (META) were obtained from TAU3AAG medium as previously described (Contreras et al. 1985, Bonaldo et al. 1988). Intracellular derived metacyclic amastigotes (IMA) were purified from monolayer of Vero cells $72 \mathrm{~h}$ after infected with metacyclic trypomastigotes. Infected monolayers were gently tripsynised, and the detached cells were recovered by centrifugation and re-suspension in PBS $(0.15 \mathrm{M}$ sodium chloride, $0.02 \mathrm{M}$ sodium phosphate, $\mathrm{pH}$ 7.2) supplemented with $1 \%$ BSA (bovine serum albumin) (PBS-BSA). A 10 $\mathrm{ml}$ aliquot of the re-suspension was disrupted by passage through a 25 -gauge needle (Piras et al. 1982). The intracellular amastigotes were separated from the cell de- bris by centrifugation in a $15-21 \%$ discontinuous Metrizamide gradient (Carvalho \& De Souza 1983). Extracellular derived metacyclic amastigotes (EMA) were obtained from metacyclic trypomastigotes incubated in MEMTAU medium as described below. META, IMA and EMA were washed twice with PBS, concentrated by centrifugation in Eppendorff tubes $\left(12,000 \mathrm{xg}, 5 \mathrm{~min} 4^{\circ} \mathrm{C}\right)$. Wet masses of parasites $(20-25 \mathrm{mg})$ were stored at $-70^{\circ} \mathrm{C}$ until use.

In vitro amastigogenesis - DEAE-52-purified metacyclic trypomastigotes (PSG: $0.073 \mathrm{M} \mathrm{NaCl} ; 0.005 \mathrm{M}$ sodium phosphate; $1 \%$ Glucose, $\mathrm{pH} 8.0$; ionic strength (I) 0.181 ) were concentrated by centrifugation and re-suspended in cold MEM medium without serum (1.0 to $1.5 \mathrm{x}$ $10^{9}$ parasites $\left./ \mathrm{ml}\right)$. One $\mathrm{ml}$ of re-suspended parasites was transferred to sterile plastic tissue culture flasks $\left(175 \mathrm{~cm}^{2}\right.$; Falcon Labware, Oxnar, CA) containing $14 \mathrm{ml}$ of MEMTAU medium $\mathrm{pH}$ 5.8, which consists of a 1:1 mixture of TAU3AAG medium and MEM 10\% FBS medium, supplemented with $70 \mathrm{mM}$ sucrose, $20 \mu \mathrm{g} / \mathrm{ml}$ bovine or human hemoglobin, $200 \mathrm{U} / \mathrm{ml}$ penicillin, $200 \mu \mathrm{g} / \mathrm{ml}$ streptomycin, and $20 \mathrm{mM}$ MES [2 (N-morpholinoethanesulfonic) acid hydrate], followed by incubation at $37^{\circ} \mathrm{C}$ without agitation in a $5 \% \mathrm{CO}_{2}$ atmosphere for three days as the preincubation phase. After three days of pre-incubation, the parasites were centrifuged and re-suspended in PSG $(\mathrm{I}=0.145)$, washed once, and applied to the top of a DEAE52 (diethylaminoethyl) column previously equilibrated with PSG (I = 0.145). Parasites were eluted with $20 \mathrm{ml}$ of PSG $(I=0.145)$ at room temperature. Aliquots of eluates containing $100 \%$ metacyclic trypomastigotes were concentrated by centrifugation $\left(8,000 \mathrm{xg}, 10 \mathrm{~min}\right.$, and $\left.4^{\circ} \mathrm{C}\right)$ and re-suspended in fresh MEMTAU medium. Parasite suspensions were transferred to sterile culture flasks containing $15 \mathrm{ml}$ of MEMTAU medium (final concentration of $\sim 4 \times 10^{7}$ forms $/ \mathrm{ml}$ ), followed by incubation at $37^{\circ} \mathrm{C}$ (reincubation phase) for different times. The in vitro differentiation was monitored by counting cells in a hematocytometer and by differential counting in Giemsastained smears. The protocols performed in experiments to study the effect of temperature or incubation times are described in the legends of the Table and in Fig. 2. For mass production of EMA $(\sim 100 \%)$, parasites re-incubated for three days were centrifuged as described, and differentiated forms were dispersed in the same volume of fresh MEMTAU medium and incubated at $37^{\circ} \mathrm{C}$ for $48 \mathrm{~h}$.

Scanning electron microscopy (SEM) - Sample processing was carried out essentially as described by Andrews et al. (1987) using glass cover slips precoated with $10 \mu \mathrm{g} / \mathrm{ml}$ poly-L-lysine. Glutaraldehyde-fixed parasites were post-fixed with $1 \% \mathrm{OsO}_{4}$ in sodium cacodylate buffer, dehydrated in graded ethanol and critically point dried from liquid $\mathrm{CO}_{2}$ without the use of a transition fluid. Specimens were coated with gold-palladium in a BALTEC SCD050 evaporator before being examined in a Philips XL-20 scanning electron microscopy.

Lysis of parasites - Wet masses of parasites were suspended $(25 \mathrm{mg} / 100 \mu \mathrm{l})$ in lysis solution $(150 \mathrm{mM} \mathrm{NaCl}$, $10 \mathrm{mM}$ Tris/HCl pH 7.4, 1 mM EDTA, 1 mM Iodoacetamide, $1 \mathrm{mM}$ 1,10-phenanthroline, $0.1 \mathrm{mM}$ PMSF (phenylmethylsulfonyl fluoride), $0.5 \mathrm{mM}$ TLCK (tosyllysylchloromethylketone), $0.5 \mathrm{mM}$ TPCK (tosylphenyl- 
alanylchloromethylketone), $0.1 \mathrm{mM}$ E-64 [L-transepoxysuccinylleucyl-amido(4-guanidino)-butane], $25 \mu \mathrm{M}$ leupeptin, $10 \mu \mathrm{g} / \mathrm{ml}$ antipain, $0.5 \% \mathrm{NP}-40$ ), frozen quickly in dry ice-ethanol, thawed in a $37^{\circ} \mathrm{C}$ water bath and vigorously vortexed for $3 \mathrm{~min}$ at room temperature. After three cycles of freezing and thawing, the disrupted parasites were centrifuged at $12,000 \mathrm{~g}$ for $5 \mathrm{~min}$. The supernatants were collected and stored in aliquots, which were either used immediately or frozen for storage at $-70^{\circ} \mathrm{C}$ in $5 \%$ glycerol. The protein contents of the supernatants were quantified using the Coomassie Plus assay (Pierce, Rockford, Illinois).

Analysis of polypeptides and glycopeptides by sodium dodecylsulfate-polyacrylamide gel electrophoresis (SDS-PAGE) - The proteins were analysed by electrophoresis on a linear gradient of $7-15 \%$ polyacrylamide gels (Laemmli 1970). Wells were loaded with equivalent amounts of protein: $12 \mu \mathrm{g}$ for polypeptides, and $20 \mu \mathrm{g}$ for glycopeptides. The resulting protein patterns were stained with a combined Coomassie Blue-Silver staining procedure (De Moreno et al. 1985) for proteins, and combined Periodic Acid-Alcian Blue-Glutaraldehyde-Silver (PAABGS) stain procedure for glycoproteins. Oxidation with periodic acid and subsequent Alcian Blue prestaining was performed according to Moller and Poulsen protocol (Moller et al. 1993, 1995). Silver staining of glycoproteins and reversal silver staining of proteins in the polyacrylamide gel was achieved by treating the gel with glutaraldehyde. The method of Dubray and Bezard (1982) was used except that 5\% aqueous Glutaraldehyde solution was employed instead of $10 \%$ Glutaraldehyde buffered. The gel was stained at room temperature for $45 \mathrm{~min}$ with $0.1 \%$ aqueous silver nitrate rather than $0.4 \%$ aqueous silver nitrate at $40^{\circ} \mathrm{C}$ for $6.5 \mathrm{~min}$. All the steps were carried out with mild agitation. Two types of markers were run in the same gel: the wide molecular weight range used to estimate molecular weight and to control the reversal silver staining (unstaining) of proteins; and a mixture $(1 \mu \mathrm{g} / \mu \mathrm{l})$ of proteoglycans and glycoproteins $(6 \mu \mathrm{l} /$ well). The following glycoconjugates were used: Chondroitin 6-Sulfate from shark cartilage (type C, Sigma C-4384), Mucin from bovine sub-maxillary glands (type I-S, Sigma M-3895), Fetuin from fetal calf serum (Sigma F-2379), and Bovine $\alpha$ Acid Glycoprotein (orosomucoid, Sigma G-9014). The gels were then scanned in a Bio-Rad Imaging Densitometer, model GS-690, and their profiles were analysed using the Bio-Rad Molecular Analyst ${ }^{\circledR} / \mathrm{PC} 1.2$ software package.

Antisera production and Western blot analysis Metacyclic trypomastigote stage-specific antiserum (antiMETA) was prepared as previously described (Contreras et al. 1998). Antiserum directed against intracellular metacyclic-derived amastigotes (anti-IMA) was prepared in New Zealand rabbits as follows. Twenty mg (wet weight) of parasites was suspended in $2 \mathrm{ml}$ of Freud's complete adjuvant and injected in rabbits in intervals of 8 days, first subcutaneously in the back, and then twice intramuscularly. After two weeks, the rabbits were inoculated intravenously three times at $48 \mathrm{~h}$ intervals with $2 \mathrm{mg}$ of freeze-thawed parasites in saline solution. Two weeks after the last inoculation, the immune response was assayed by indirect immunofluorescense with the homolo- gous antigen. Three or four weeks after the last inoculation, the rabbits were bled and the antiserum obtained was kept in aliquots at $-70^{\circ} \mathrm{C}$.

For Western blot analyses, total proteins of META, IMA and EMA were electrophoretically transferred from a linear gradient of 7-15\% SDS-PAGE gels to nitrocellulose membranes according to the method of Towbin et al. (1979) as previously described (Contreras et al. 1998). Four $\mu \mathrm{g}$ of protein of each stage were applied to the lanes. The membranes were blocked and incubated overnight at $4{ }^{\circ} \mathrm{C}$ with a 1: 8,000 dilution of anti-META serum or 1:5,000 dilution of anti-IMA serum. The antigens were revealed using peroxidase-conjugate goat anti-rabbit total immunoglobulins $(1: 10,000)$ and the Luminol chemiluminescense system according to the protocol supplied by the manufacturer (Super-Signal, Pierce). Finally, the blots were wrapped in plastic wrap and luminographed using AGFA films. The immunoblots were then scanned and their profiles were analysed as described above.

\section{RESULTS}

In vitro transformation of metacyclic trypomastigotes into amastigotes - At $37^{\circ} \mathrm{C}$, MEM $0.4 \%$ BSA medium has been reported to induce $100 \%$ of bloodstream trypomastigotes into amastigotes (Tomlinson et al. 1995). However, under these conditions metacyclic trypomastigotes failed to produce amastigotes, and after one day of incubation all the parasites lysed. After testing different media and incubation conditions, we could obtain a high yield of differentiation forms and EMA at $37^{\circ} \mathrm{C}$ in a $5 \% \mathrm{CO}_{2}$ atmosphere. This was achieved in two steps: Step 1 consisted of the pre-incubation of metacyclic trypomastigotes at $37^{\circ} \mathrm{C}$ in MEMTAU for three days. The metacyclic trypomastigotes underwent very few morphological changes, and a high percentage of well-preserved metacyclic trypomastigotes (up 80\%) was obtained; Step 2: the subpopulation of untransformed metacyclic trypomastigotes was purified through DEAE-52 columns $(I=0.145)$, and when these purified metacyclic trypomastigotes were incubated again in fresh MEMTAU medium at $37^{\circ} \mathrm{C}$, a high percentage (up 80\%) of typical, well-preserved amastigotes were progressively produced. The in vitro-derived amastigotes did not display any detectable signs of degeneration and were indistinguishable from normal amastigotes.

In vitro generated amastigotes are morphologically indistinguishable from naturally produced amastigotes - Fig. 1 shows the morphology of metacyclic forms and compares EMA and IMA by SEM. Metacyclic trypomastigotes that had recently been transformed from epimastigotes and purified through a DEAE-52 column (I $=0.181$ ) showed a slender morphology and displayed a subterminal kinetoplast (arrow, Fig. 1A). General aspects of the EMA and IMA are shown in Fig. 1B, C, respectively. The comparison of EMA and IMA isolated by Metrizamide gradient showed a remarkable morphological similarity. They were rounded or oval-shaped and a short protruding flagellum was observed. Further characterization of the transformation process will be given elsewhere (Contreras et al., in preparation). 
Effect of temperature, pre-incubation and incubation and age of the culture media on the in vitro production of amastigotes from metacyclic trypomastigotes - We wanted to test which other factors affected the in vitro transformation process. When metacyclic trypomastigotes were pre-incubated at $37^{\circ} \mathrm{C}$, purified by DEAE52 columns, and incubated in fresh medium at $37^{\circ} \mathrm{C}$ a high percentage of amastigotes $(85 \pm 6 \%)$ was observed (Table). However, when they were pre-incubated at a lower temperature $\left(27^{\circ} \mathrm{C}\right)$, purified and placed at $37^{\circ} \mathrm{C}$, only $25 \pm 5 \%$
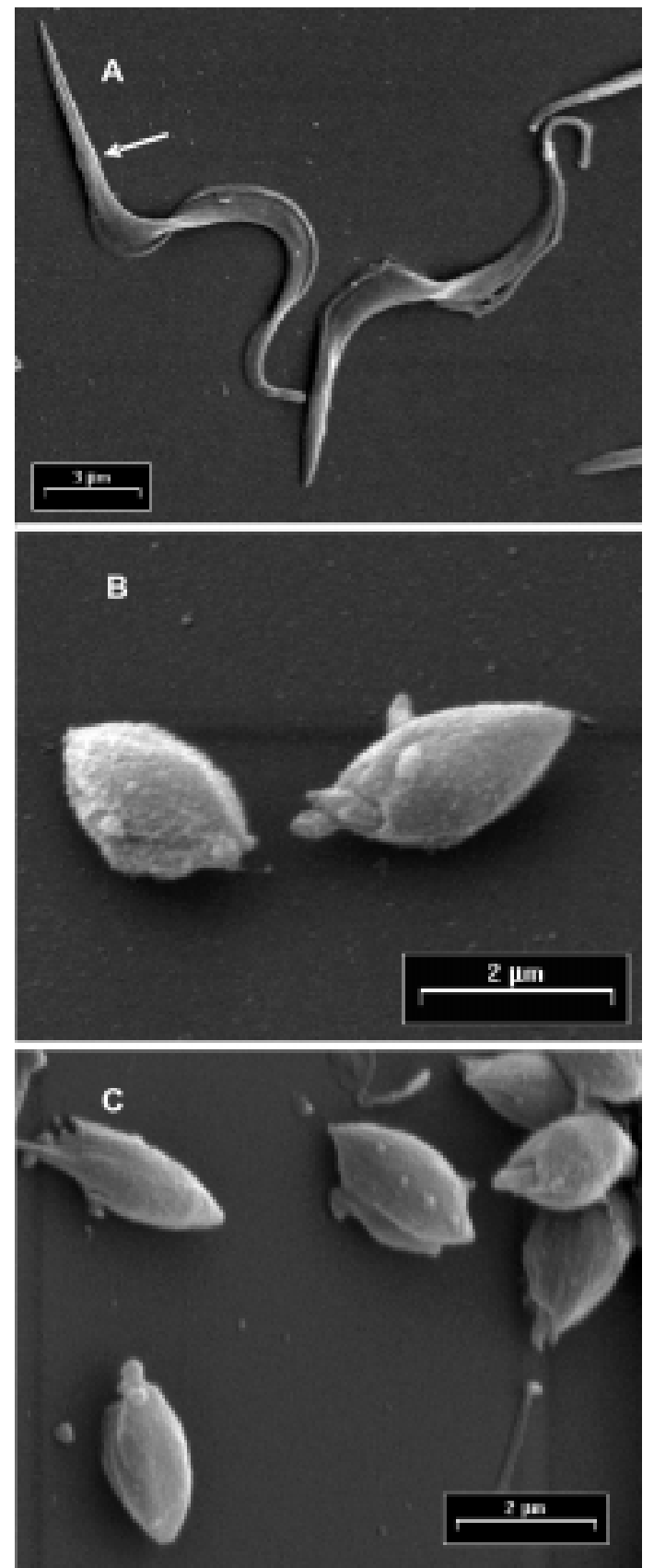

Fig. 1: scanning electron micrograph of various stages of Trypanosoma cruzi (EPm6). A: metacyclic trypomastigotes induced in TAU3AAG medium and chromatographed through DEAE-cellulose columns; B: intracellular metacyclic-derived amastigotes obtained by disruption of infected Vero cells; C: extracellular metacyclic-derived amastigotes induced in MEMTAU medium. of amastigotes was observed. In addition, incubation of metacyclic trypomastigotes in fresh medium at $27^{\circ} \mathrm{C}$, followed by purification and incubated at the same temperature $\left(27^{\circ} \mathrm{C}\right)$ did not affect parasite morphology $(100 \%$ metacyclic trypomastigotes). On the other hand, when temperature-committed metacyclic trypomastigotes were incubated in fresh medium at $27^{\circ} \mathrm{C}$ for 3 days, the pattern of morphologic changes showed a higher percentage of differentiation forms $(38 \pm 7 \%)$ than amastigotes $(10 \pm$ $4 \%$ ), indicating that this metacyclic trypomastigote population could not complete the morphological transformation at the lower temperature.

Purification of parasites through DEAE-52 had no effect on committing metacyclic trypomastigotes to amastigogenesis. Parasites that were pre-incubated for three days at $37^{\circ} \mathrm{C}$ and then transferred to fresh media without passing through DEAE-52 columns were still capable of producing large numbers of amastigotes $(80 \pm$ $3 \%$, Table ). It is therefore unlikely that the purification step modulates the parasite's ability to undergo amastigogenesis in any significant way.

Finally, the variables of parasite titer, volume and quality of the incubation medium were tested. Modifications in the inoculation titer ranging from $5 \times 10^{6}$ to $7 \times 10^{7}$ parasites $/ \mathrm{ml} ; \mathrm{pH}$ (5.3 to 6.9) and the depth of medium (1 to $7 \mathrm{~mm}$ ) had no significant effect on the transformation process (data not shown). However, we noticed that amastigotes production is markedly compromised in old medium. For instance, if the same pre-incubation medium used to "pre-condition" the metacyclic trypomastigotes is used again for incubation, a fairly low number of amastigotes were obtained ( $57 \pm 6 \%$, Table ) in comparison with parasites incubated in fresh medium. In all likelihood, this may be due to the progressive exhaustion of nutrients and/or catabolite accumulation in the media.

During the course of experimentation, it became evident that temperature $\left(37^{\circ} \mathrm{C}\right)$ is crucial to allowing metacyclic trypomastigotes to become competent and complete the amastigogenesis process. In parasite-vertebrate cell culture systems, it is known that following penetration, metacyclic trypomastigotes differentiate into amastigotes in approximately $3 \mathrm{~h}$ and remain morphologically quiescent for about $35 \mathrm{~h}\left(\right.$ at $\left.35^{\circ} \mathrm{C}\right)$ prior to the onset of reproduction (Dvorak 1975). If the observed temperature-induced transformation reported here has physiological significance, pre-incubation at $37^{\circ} \mathrm{C}$ for different times should affect the number of metacyclic trypomastigotes committing to amastigogenesis. To test this hypothesis, metacyclic trypomastigotes were pre-incubated at $37^{\circ} \mathrm{C}$ for 1, 2 and 3 days. The parasites were later purified and incubated in fresh MEMTAU medium. Their respective transformation kinetics were measured during the following six days of culture. Metacyclic trypomastigotes incubated for six days at $37^{\circ} \mathrm{C}$ without pre-incubation were used as control.

Fig. 2 shows the effect of pre-incubation length on the capacity of metacyclic trypomastigotes to transform into amastigotes. The transformation process was accelerated when the metacyclic trypomastigotes were pre-incubated for 1, 2 or 3 days. Parasites pre-incubated for a single day yielded $51 \pm 1 \%$ and $78 \pm 5 \%$ of amastigotes after three or five days of incubation, respectively. When 
TABLE

Effect of the pre-incubation and incubation temperature on the capacity of Trypanosoma cruzi metacyclic trypomastigotes to transform into amastigotes in MEMTAU medium

\begin{tabular}{|c|c|c|c|c|}
\hline \multicolumn{2}{|c|}{ Temperature $^{a}$} & \multicolumn{3}{|c|}{ Percentages of forms $(\mathrm{X} \pm \mathrm{SD})$} \\
\hline Pre-incubation & Incubation & Metacyclic & Differentiation & Amastigotes \\
\hline $37^{\circ} \mathrm{C}$ & $37^{\circ} \mathrm{C}$ & $5 \pm 2$ & $10 \pm 3$ & $85 \pm 6$ \\
\hline $27^{\circ} \mathrm{C}$ & $37^{\circ} \mathrm{C}$ & $59 \pm 4$ & $16 \pm 5$ & $25 \pm 3$ \\
\hline $27^{\circ} \mathrm{C}$ & $27^{\circ} \mathrm{C}$ & 100 & 0 & 0 \\
\hline $37^{\circ} \mathrm{C}$ & $27^{\circ} \mathrm{C}$ & $52 \pm 6$ & $38 \pm 7$ & $10 \pm 4$ \\
\hline $37^{\circ} \mathrm{C}^{b}$ & $37^{\circ} \mathrm{C}$ & $3 \pm 1$ & $17 \pm 4$ & $80 \pm 3$ \\
\hline $37^{\circ} \mathrm{C}^{c}$ & $37^{\circ} \mathrm{C}$ & $28 \pm 4$ & $15 \pm 5$ & $57 \pm 6$ \\
\hline
\end{tabular}

$a$ : metacyclic trypomastigotes from TAU3AAG medium were pre-incubated for 3 days. Untransformed metacyclic trypomastigotes were purified through DEAE-cellulose columns $(\mathrm{I}=0.145)$ and incubated for 3 days as described in Materials and Methods; $b$ : parasites incubated without previous purification through of DEAE-cellulose column; $c$ : parasites incubated in the used preincubation medium (older). Data correspond at least two different experiment duplicated.

pre-incubated for two days, amastigotes were generated at a yield of $86 \pm 4 \%$ and $77 \pm 6 \%$ at the end of days three and four of incubation. Finally, if the parasites were preincubated for 3 days, the percentage of amastigotes rapidly increased to $85 \pm 6 \%$ toward the end of the third day of incubation. It is important to note that the control condition (not pre-incubation) showed a steady increase in the percentage of amastigotes produced $(14 \pm 3 \%$ and

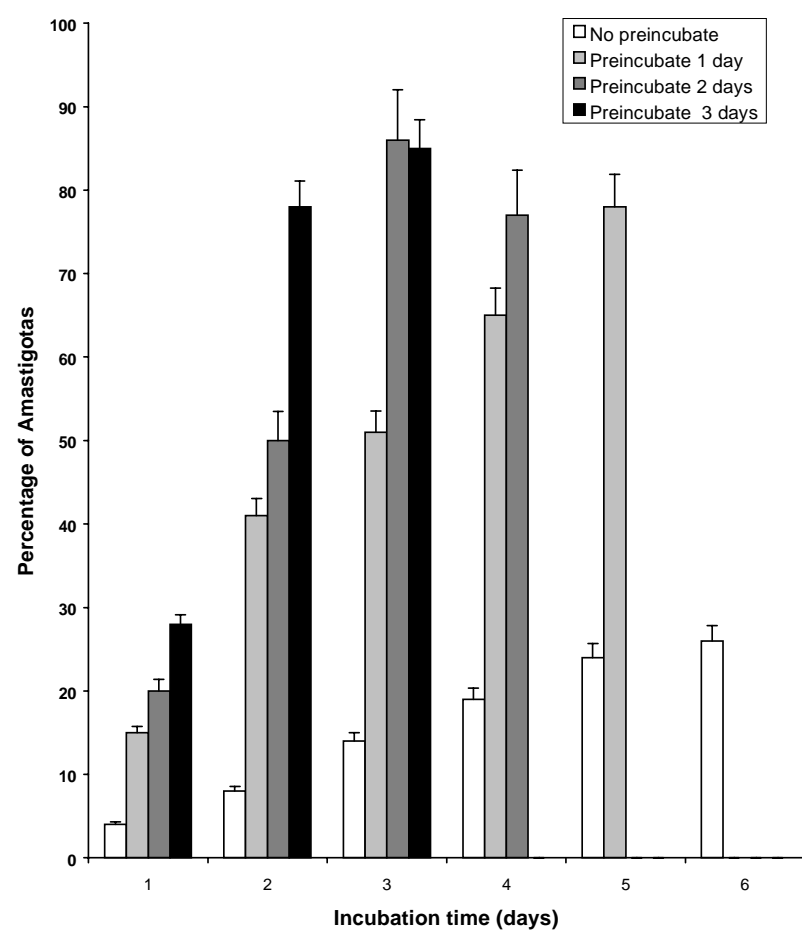

Fig. 2: effect of pre-incubation and incubation time on the capacity of metacyclic trypomastigotes to transform into amastigotes. All parasites were raised in MEMTAU medium at $37^{\circ} \mathrm{C}$. Untransformed metacyclic trypomastigotes were pre-incubated for 1, 2 and 3 days, purified through DEAE-cellulose columns $(I=0.145)$, and incubated for 6 days as described in Materials and Methods. Metacyclic trypomastigotes (no pre-incubated) incubated for 6 days at $37^{\circ} \mathrm{C}$ were used as control. Only the percentages of amastigotes obtained are reported here. Data correspond to at least two different experiments each carried out in duplicate.
$26 \pm 3 \%$ during days 3 and 6 , respectively), but never reached the numbers obtained in the pre-incubation experiments. These results indicate that pre-incubation at $37^{\circ} \mathrm{C}$ induces a commitment of metacyclic trypomastigotes to differentiation and indicates that under our experimental conditions (without steady-state) pre-incubation for two days was sufficient to produce large number of amastigotes.

Thus, the data on culture conditions suggest that in vitro amastigogenesis occurs in two phases: a temperature-dependent induction of transformation, and an initiation of morphological transformation which is dependent on temperature and the availability of fresh, proteinrich medium.

In vitro generated amastigotes show a high degree of similarity of polypeptides and glycopeptides with the naturally produced amastigotes - With the purpose of making a molecular-level comparison of the stages produced, total proteins from META, EMA and IMA were analyzed by SDS-PAGE, and their polypeptide profiles were visualized by Coomassie Blue-Silver as shown in Fig. 3A. Two distinct protein profiles can be observed. One displayed by TAU3AAG-purified metacyclic trypomastigotes before incubation in MEMTAU medium at $37^{\circ} \mathrm{C}$ (lane META, Fig. 3A) and another shared by both amastigotes (EMA and IMA). It is worth noting that EMA displayed a protein profile significantly different to that of META, and that the profiles of IMA and EMA show surprising similarities between the 205 and $14.2 \mathrm{kDa}$ markers.

To determine whether there were changes at the glycoprotein level, as observed in the protein profiles, an equivalent gel was visualised by the PAABGS procedure as shown in Fig. 3B. The patterns of glycopeptides obtained are characteristic for each morphological stage. Metacyclic trypomastigotes showed a pattern characterized by a major glycopeptide band with a molecular mass ranking from 80 to $92 \mathrm{kDa}$, and the presence of a smear of glycopeptides with a molecular mass ranking from 47 to $57 \mathrm{kDa}$ (white triangles at left of lane META, Fig. 3B). The comparison of this profile with the glycoprotein profile displayed by EMA shows significant changes, represented by the appearance of an intensely silver-stained 

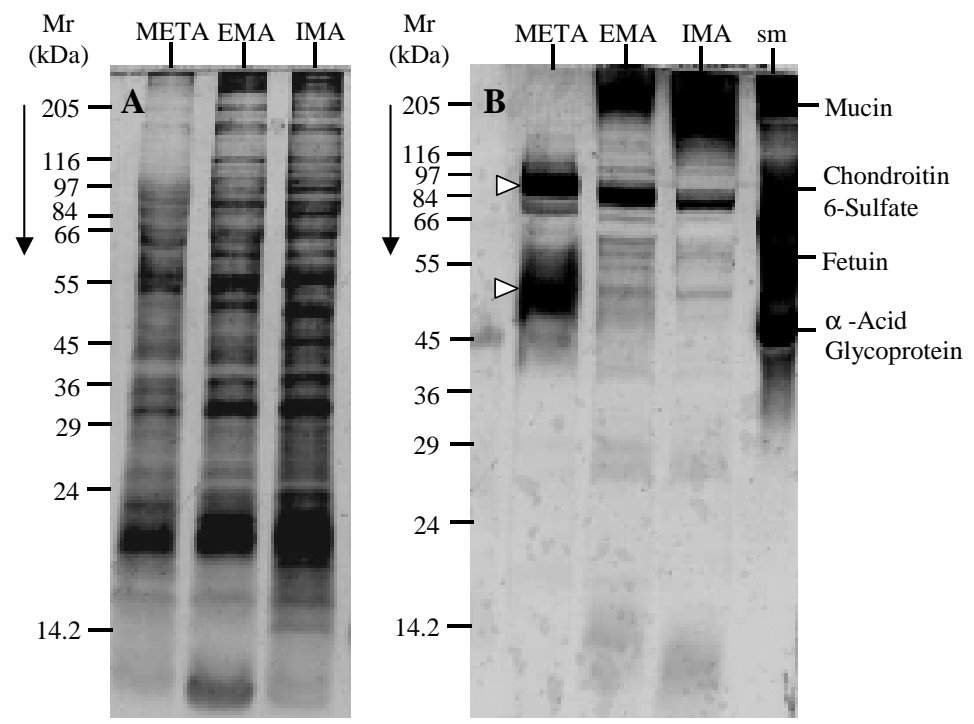

Fig. 3: SDS-PAGE analyses of different stages of Trypanosoma cruzi (EPm6) proteins. A: $12 \mu \mathrm{g} / \mathrm{well}$ and glycoproteins; B: $20 \mu \mathrm{g} / \mathrm{well}$. META refers to metacyclic trypomastigotes raised in TAU3AAG medium; IMA refers to intracellularly-derived metacyclic amastigotes; and EMA to extracellular metacyclic-derived amastigotes. Mr correspond to relative mobility of the markers in kilodaltons (kDa). Lane "sm" was loaded with $6 \mu \mathrm{g}$ of a mixture of glycoconjugates for assaying the glycoprotein staining procedure. The position of glycoconjugates markers is shown on the right side of panel B. The gels consist of $10 \%$ uniform polyacrylamide and were stained with a combined Coomassie Blue-Silver stain procedure for proteins (A), or with a combined Periodic Acid-Alcian Blue-Glutaraldehyde-Silver (PAABGS) stain procedure for glycoproteins (B). Both procedures are described in Materials and Methods.

cluster of high molecular weight, unresolved glycopeptides migrating as the Mucin marker, which is absent in META, a rearrangement of glycopeptides between the 66 and $97 \mathrm{kDa}$ markers with a major glycopeptide band of molecular mass ranking from 75 to $85 \mathrm{kDa}$ and the appearance of at least 5 well-resolved glycopeptides of similar intensity between the 66 and $45 \mathrm{kDa}$ markers. A comparison of EMA and IMA patterns shows remarkable similarities. These results indicate that the morphological transformation of the parasites is associated with specific changes in their polypeptides and glycopeptides profiles and may reflect the degree of similarity of protein content shared by both types of amastigotes.

Western blot analysis of stage-shared antigens Stage-specific antigens were used to determine if the changes observed in META, EMA and IMA proteins and glycoprotein profiles reflected specific morphological changes. Western blots of all three stages were performed using stage-specific antisera. Fig. 4A shows antigen profiles of META, EMA and IMA revealed by anti-META serum. Metacyclic trypomastigotes showed a pattern characterized by two dark clusters of unresolved antigens, a smear $(95 / 80,43 / 33$ and 52/46 kDa, respectively, white triangles at left of lane META, Fig. 4A) and five barely detectable antigens $(66,64,60,45$ and $25 \mathrm{kDa}$, short bars at left of lane META, Fig. 4A). The comparison of this profile and the one displayed by EMA shows significant changes represented by the appearance of at least four antigenic bands (> 175 cluster, 115,58 and $43 \mathrm{kDa}$, short bars at the left of lane EMA, Fig. 4A) which are absent in META. Four antigenic bands were recognized by antiMETA serum in META, EMA and IMA (82, 64,52 and 47 $\mathrm{kDa}$, short bars at the left of lane IMA, Fig. 4A). The comparison of EMA and IMA patterns shows similarities
(Fig. 4A). Finally, the antigenic profiles of META, EMA and IMA revealed by anti-META serum (Fig. 4A) resemble the glycopeptide profiles of these stages (Fig. 3B), suggesting that almost all of the glycosilated molecules of the metacyclic trypomastigotes are highly immunogenic.

Stage comparisons using anti-IMA serum confirmed the remarkable similarity between both amastigotes studied (EMA and IMA, Fig. 4B), which share at least eight antigenic bands of different width and density $(>175,75$, $72,70,64,60,55$ and $51 \mathrm{kDa}$, short bars at the left of lane EMA, Fig. 4B). Likewise, META was poorly recognized by anti-IMA (Fig. 4B). It should be noted that the $47 \mathrm{kDa}$ and cluster $12 / 5 \mathrm{kDa}$ and several antigenic bands (135, 120,105 and $95 \mathrm{kDa}$ ) in the region of the $116 \mathrm{kDa}$ marker revealed by anti-IMA in IMA (short bars at left of lane IMA, Fig. 4B) were not present in the other two stages, suggesting that such antigens may be IMA-specific.

Therefore, the immunoblotting data indicate that the morphological transformation of the parasites are most likely associated with antigenic changes and are likely to reflect stage-specific molecular profiles. Similarly, these data also suggest that common antigens are shared by metacyclic and amastigotes stages.

\section{DISCUSSION}

One of the main findings of the present study is that amastigogenesis can be triggered in vitro by temperature changes in media supplemented with proteins (serum). The practical extension of this result being the development of a system that allows the in vitro production of high yields of extracellular T. cruzi amastigotes from metacyclic trypomastigotes. Most of the information about factors triggering in vitro amastigogenesis has been obtained by assaying bloodstream trypomastigotes in which the para- 


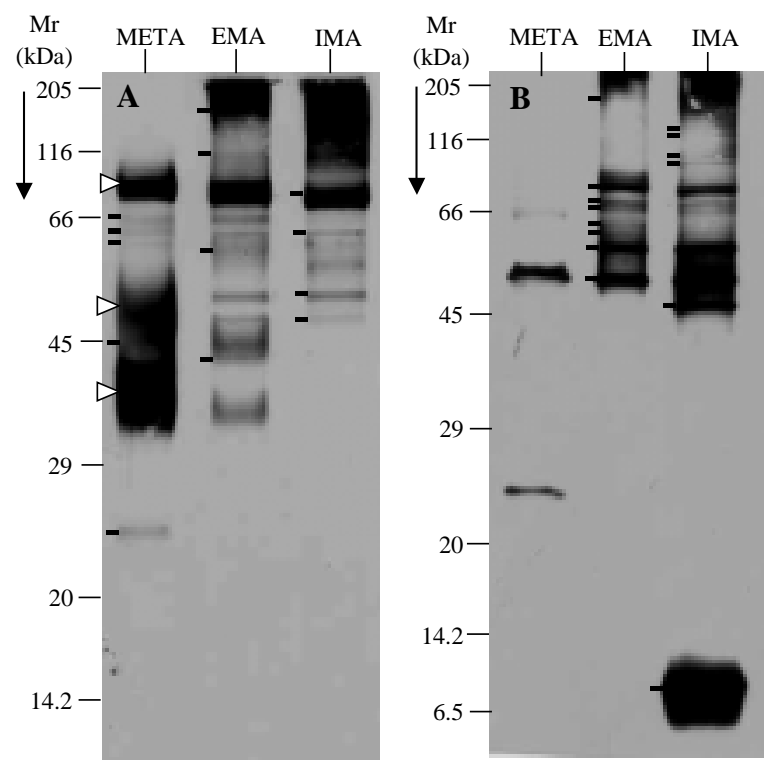

Fig. 4: Western blot analyses of different stages of Trypanosoma cruzi (EPm6) using anti-metacyclic trypomastigote serum (anti-META, A) and anti-intracellularly-derived metacyclic amastigote serum (anti-IMA, B) as described in Materials and Methods. META refers to metacyclic trypomastigotes raised in TAU3AAG medium $(4 \mu \mathrm{g})$; EMA to extracellular metacyclic-derived amastigotes $(4 \mu \mathrm{g})$ and IMA to intracellularly-derived metacyclic amastigotes $(4 \mu \mathrm{g})$. Mr correspond to relative mobility of the markers in kilodaltons (kDa).

sites are neither subjected to a heat shock nor to density changes in their environment. It is known, for instance, that low $\mathrm{pH}$ accelerates the extracellular transformation of tissue culture-derived trypomastigotes into amastigotes (Kambara et al. 1990, Tomlinson et al. 1995). However, metacyclic trypomastigotes incubated under identical conditions do not differentiate but rather undergoes cell death over a 24-hour period (Tyler \& Engman 2001). This fact seems to indicate that metacyclic trypomastigotes posses a specific way of responding and adapting to environmental changes that is different to that shown by bloodstream trypomastigotes and may explain the previous inability to produce amastigotes axenically.

Several authors have devised cell-free media in which a mixture of epimastigotes and metacyclic trypomastigotes from cultures of $T$. cruzi are induced to transform into amastigotes (Pan 1978, Kimura et al. 1978, Villalta \& Kierzenbaum 1982, Rondinelli et al. 1988). In such assays, the amastigotes were gradually selected by employing highly enriched media and a progressive increase of the incubation temperature by serial culture at $35.5^{\circ} \mathrm{C}$ or $37^{\circ} \mathrm{C}$. The approach described in this report does away with the use of developmentally heterogeneous populations of parasites to produce amastigotes. Instead, it relies on a homogeneous, purified metacyclic trypomastigote population raised in a chemically-defined medium at $27^{\circ} \mathrm{C}$. Preincubation of this axenic metacyclic population for at least two days at $37^{\circ} \mathrm{C}$ in a protein-supplemented medium becomes amastigogenic when cultured again in fresh, protein-rich medium (Fig. 2). Furthermore, the addition of a DEAE-cellulose purification step to remove differentiation forms and enrich metacyclics after pre-incubation did not affect their ability to undergo amastigogenesis.

We can not rule out the possibility that specific proteins present in sera might act as triggers for the differentiation process. However, parasites pre-incubated in me- dia (without serum) and supplemented with hydrolyzed lacto albumin and incubated in MEMTAU medium also underwent the reported transformation process, although to a lesser extent (data not shown). We therefore believe that the "pre-conditioning" serves to exert physical changes on the purified metacyclic trypomastigote, resulting in measurable intrinsic changes in the biological properties of the parasite. Future experiments will test whether culturing the parasites in media of different specific densities is sufficient to trigger amastigogenesis on its own.

We also demonstrate that the EMA produced in vitro are similar to IMA using several different criteria (gross morphology, protein and glycoprotein profiles as well as immunogenic profiles). In fact, preliminary data show that the in vitro produced EMAs are not only capable of infecting cultured cells and mice, but are also resistant to macrophage digestion (data not shown). Recently, we have studied the changes that occur at molecular level during the in vitro metacyclic trypomastigote-amastigote transformation. We founded that the in vitro primary amastigogenesis is associated with abrupt changes in protein, glycoprotein, and stage-specific antigens that occur simultaneously during the first $24 \mathrm{~h}$ of pre-incubation (Contreras et al. 2002).

Therefore, based on the results reported here, we postulate that at least two factors are involved in triggering primary amastigogenesis: the increase in temperature and the changes in viscosity encountered by metacyclic trypomastigotes upon invasion of the vertebrate host. It has been reported that the heat-shock proteins (hsp) could play an important role in the infection and differentiation of T. cruzi (Requena et al. 1992). However, the effect that the rheologycal properties of the transmission fluids may play in the T. cruzi life-cycle have yet to be systematically studied and should be explored more extensively. 
In conclusion, this paper reports for the first time the in vitro production of extracellular amastigotes from axenically-reared metacyclic trypomastigotes. It is still premature to assume that our in vitro differentiation system precisely recapitulates naturally occurring amastigogenesis, i.e., the transformation of metacyclic trypomastigotes from triatomine urine into amastigotes inside the cells of mammalian hosts. However, preliminary data indicate that in vitro produced amastigotes cannot be distinguished from intracellular, metacyclic-derived amastigotes obtained from Vero cells in terms of their biological properties (Contreras et al., in preparation). One clear advantage of our experimental system is that it makes use of in vitro produced metacyclic trypomastigotes, which can be massively obtained from chemically defined media. Thus, EMA obtained in vitro could serve to delineate the molecular differences and similarities that must exist between amastigotes and trypomastigote-derived amastigotes, effectively providing a simple and inexpensive alternative for the identification and testing of new chemotherapeutic and immune prophylactic agents.

\section{ACKNOWLEDGEMENTS}

To Dr Alejandro Sánchez for his critical suggestions and helpful discussion of the manuscript. To Gregorio Flores and Mr Wilmer Pineda for their excellent technical assistance.

\section{REFERENCES}

Andrews NW, Hong K, Robbins ES, Nussenzweig V 1987. Stage-specific surface antigens expressed during the morphogenesis of vertebrate forms of Trypanosoma cruzi. Exp Parasitol 64: 1-11.

Bonaldo MC, Souto-Padron T, Sousa W, Goldenberg S 1998. Cell-substrate adhesion during Trypanosoma cruzi differentiation. J Cell Biol 106: 1349-1382.

Brener Z 1973. Biology of Trypanosoma cruzi. Ann Rev Microbiol 27: 347-382.

Burgleigh B, Andrews N 1995. The mechanism of Trypanosoma cruzi invasion of mammalian cells. Ann Rev Microbiol 49: 175-200.

Carvalho U, De Sousa W 1983. Separation of amastigotes and trypomastigotes of Trypanosoma cruzi from culture cells. Z Parasitenkd 69: 571-575.

Chiari E 1975. Differentiation of Trypanosoma cruzi in culture. In New Approaches in American Trypanosomiasis Research. PAHO Sci Publ 318: 144-145.

Contreras VT, Araque W, Delgado V 1994. Trypanosoma cruzi: metacyclogenesis in vitro. I. Changes in the properties of metacyclic trypomastigotes maintained in the laboratory by different methods. Mem Inst Oswaldo Cruz 89: 253-259.

Contreras VT, De Lima AR, Zorrilla G 1998. Trypanosoma cruzi: maintenance in culture modify gene and antigenic expression of metacyclic trypomastigotes. Mem Inst Oswaldo Cruz, 93: 753-760.

Contreras VT, Navarro MC, De Lima AR, Duran F, Arteaga R, Franco Y 2002. Early and late molecular and morphologic changes that occur during the in vitro transformation of Trypanosoma cruzi metacyclic trypomastigotes to amastigotes. Biol Res 35: 21-32.

Contreras V, Salles J, Thomas N, Morel C, Goldenberg S 1985. In vitro differentiation of Trypanosoma cruzi under chemically defined conditions. Mol Biochem Parasitol 16: 123133.

De Moreno M, Smith J, Smith R 1985. Silver staining of pro- teins in polyacrilamide gels: increased sensitivity trough a combined blue-silver stain procedure. Anal Biochem 151: 466-470.

Deane MP, Moriearty PL, Thomaz N 1984. Cell differentiation in trypanosomatids and other parasitic protozoa. In CM Morel, Genes and Antigens of Parasites. A Laboratory Manual, 2nd ed., Fundação Oswaldo Cruz, Rio de Janeiro, p. 11-21.

Dubray G, Bezard G 1982. A highly sensitivity periodic acidsilver stain for 1,2-dol groups of glycoproteins and polysaccharides in polyacrilamide gels. Anal Biochem 119: 325329.

Dvorak JA 1975. New in vitro approach to quantitation of Trypanosoma cruzi-vertebrate cell interaction. In New Approaches in American Trypanosomiasis Research. PAHO Sci Publ 318: 109-145.

Kambara H, Uemura H, Nakazawa S, Fukama T 1990. Effect of low $\mathrm{pH}$ on transformation of Trypanosoma cruzi trypomastigote to amastigote. Japan J Parasitol 39: 226228.

Kimura E, Lay W, Fernandez J 1978. Extracellular in vitro evolution of metacyclic trypomastigotes isolated from Trypanosoma cruzi cultures. Rev Inst Med Trop São Paulo 20: 133-138.

Laemmli UK 1970. Cleavage of structural proteins during the assembly of the head of bacteriophage T4. Nature (London) 227: 680-685.

Moller H, Heinegard D, Poulsen J 1993. Combine Alcian Blue silver stain of subnanogram quantities of proteoglycans and sulfate-polyacrylamide gels. Anal Biochem 209: 169-175.

Moller H, Heinegard D, Poulsen J 1995. Improved method for silver staining of glycoproteins in thin sodium dodecyl sulfate polyacrilamide gels. Anal Biochem 226: 371-374.

Pan C 1978. Trypanosoma cruzi: intracellular stages grown in a cell-free medium at $37^{\circ} \mathrm{C}$. Exp Parasitol 45: 215-224.

Piras MM, Piras R, Henríquez D 1982. Changes in morphology and infectivity of cell culture-derived trypomastigotes of Trypanosoma cruzi fibroblast. Mol Biochem Parasitol 6: 67-81.

Requena JM, Jimenez-Ruiz A, Soto M, Assiego R, Santarén JF, Lopez MC, Patarrollo ME, Alonso C 1992. Regulation of hsp70 expression in Trypanosoma cruzi by temperature and growth phase. Mol Biochem Parasitol 35: 201-212.

Rondinelli E, Silva R, De Oliveira F, De Almeida C, De Carvalho E, Torres F 1988. Trypanosoma cruzi: an in vitro cycle of cell differentiation in Axenic culture. Exp Parasitol 66: 197204.

Ruiz RC, Rigoni VL, Gonsalez J, Yoshida N 1993. The 35/50$\mathrm{kDa}$ surface antigen of Trypanosoma cruzi metacyclic trypomastigotes an adhesion molecule involved in host cell invasion. Parasit Immunol 15: 121-125.

Shapira M, Mc Ewan JC, Jaffe CL 1998. Temperature effects on molecular processes wich lead to stage differentiation in Leishmania. EMBO J 7: 2895-2901.

Tomlinson S, Vandekerckhove F, Frevert U, Nussenzweig V 1995. The induction of Trypanosoma cruzi trypomastigote to amastigote transformation by low $\mathrm{pH}$. Parasitology 110 : 547-554.

Towbin H, Stachelin T, Gordon J 1979. Electrophoretic transfer of protein from polyacrylamide gels to nitrocellulose sheets: Procedure and some application. PNAS 79: 43504354.

Tyler K, Engman, D 2001. The life cycle of Trypanosoma cruzi revisited. Int J Parasitol 31: 472-481.

Villalta F, Kierszenbaum F 1982. Growth of isolated Amastigotes of Trypanosoma cruzi in cell-free medium. J Protozool 29: 570-576. 